\title{
Robust B-Spline Snakes for Ultrasound Image Segmentation
}

\author{
C Tauber, H Batatia, G Morin, A Ayache \\ IRIT-ENSEEIHT, Toulouse, France
}

\begin{abstract}
Segmenting echographic images is a difficult task due to their low contrast and to the presence of speckle. This paper presents an original robust B-Spline snake based on a novel external energy. Our approach combines anisotropic diffusion with the process of curve evolution by using a local coefficient of variation and the Tukey's error norm. This makes the snake robust to speckle. At each iteration the algorithm computes an edge image, and derives a new external energy based on the amplitude and direction of the gradient of the upper mentioned coefficient. The method has been tested on echocardographic images of a 12-week old foetus. The results, presented with different parameters, show a significant improvement.
\end{abstract}

\section{Introduction}

Ultrasound (US) images are ubiquitous in cardiology. US imaging is real-time, non-radioactive, non-invasive and inexpensive. However, US imagery is characterized by low signal to noise ratio, low contrast between tissues and speckle contamination causing erroneous detection of cavities boundaries. Active contour models (snakes) deal with some of those limitations. They consider boundaries as inherently connected smooth curves. A snake is a curve that evolves from an initial position towards the boundary of an object, minimizing some energy functional $[1,2,3,4]$.

A B-spline snake is an energy minimizing spline parameterized by its control points. The smoothness of the snake is implicitly given by the B-spline model $[5,6,7,8]$. The energy consists in two terms: the internal energy and the external energy. The first affects the smoothness of the curve, and the second attracts the snake toward image features. A number of external energy terms have been proposed $[9,10,11,12,13]$. Most of these approaches either use gradient information or global image statistics. However these external energy terms cannot be used efficiently for US imaging because of the presence of speckle. A novel method for anisotropic diffusion of ultrasound images was introduced in [14]. It uses the local coefficient of variation (LCV) [15] and a robust diffusion tensor to filter echographic images. The LCV is computed locally and compared to the global coefficient of variation (GCV). In homogeneous areas affected by speckle LCV is close to GCV. Near edges LCV becomes greater. In this paper we derive a new energy term using LCV, and propose to use an internal energy term which preserves parameterization of the B-snake. The remainder of this paper is organized as follows. We first present the model for internal energy term in section 2.1, and the derivations of the new external energy term in section 2.2. In section 2.3 we present the process of curve evolution. The results are shown in section 3 .

\section{Methods}

\subsection{The internal energy model}

First, we briefly describe the classical energy based Bsplines snakes. Let $s(r):[0,1] \rightarrow \Re^{2}$ be a parametrized planar curve and let $I:[0, a] \times[0, b] \rightarrow \Re^{+}$be the image to be segmented. The snake $s$, originally introduced by Kass et al. [1], is described as an energy minimizing parametric curve guided by external $\left(E_{\text {ext }}\right)$ and internal $\left(E_{\text {int }}\right)$ forces. The minimal total energy configuration

$$
s^{*}=\arg \min _{\mathrm{s}}\left\{\mathrm{E}_{\mathrm{int}}(\mathrm{s})+\mathrm{E}_{\mathrm{ext}}(\mathrm{s}, \mathrm{I})\right\}
$$

is a compromise between smoothness and proximity to the desired image features. Schoenberg demonstrated in [16] that cubic B-splines are curves minimizing $\oint\left|s^{\prime \prime}\right|^{2}$ for a given parameterization. For this reason B-spline snakes are often considered to fullfill implicitly the smoothness requirement [7, 17, 18]. However during the curve evolution, knots can regroup toward low external energy features of the image. This affects the parameterization causing the curve to loose geometric smoothness. The best way to ensure smoothness is to use a parameterization close to the curvilinear abscissa [8]. Therefore we use an internal energy term which forces the B-spline snake to preserve its uniform parameterization :

$$
E_{\text {intern }}=\left.\oint|| s^{\prime}(u)\right|^{2}-\left.c\right|^{2} d u,
$$


where

$$
c=\frac{1}{L^{2}}(\underbrace{\left.\int_{0}^{L}\left(x^{\prime}(u)^{2}+y^{\prime}(u)^{2}\right)^{\frac{1}{2}} d u\right)^{2}}_{\text {curve length }}
$$

with $L$ the number of knots. This internal energy forces a nearly constant spacing between knots, thus, no other internal energy term is required. Tension of the curve can be controlled by increasing or decreasing the number of knots of the B-spline snake (cf section 2.3).

\subsection{Proposed external energy}

It is well known that filtering efficiently images improves the quality of segmentation. Thus the first step of our segmentation method is to filter the image with anisotropic diffusion. In [14], we proposed a robust anisotropic diffusion that uses an original diffusion tensor to effectively filter out speckle. This tensor is based on LCV and on Tukey's robust error norm. Considering LCV as an edge detector we create a new external energy term tailored to detect the heart cavities boundaries.

The anisotropic diffusion is performed by solving the following PDE :

$$
\left\{\begin{array}{c}
\frac{\delta I(x, t)}{\delta t}=\operatorname{div}[c(\operatorname{LCV}(x, t)) \nabla I(x, t)] \\
I(x, 0)=I_{0}(x),\left.\left(\frac{\delta I(x, t)}{\delta \vec{n}}\right)\right|_{\delta \Omega}=0
\end{array}\right.
$$

where div stands for the divergence, $\nabla$ denotes the gradient, $I_{0}(x)$ is the original image intensity of the pixel $x, \delta \Omega$ is the image domain border, $\vec{n}$ is the outward normal vector of $\delta \Omega$, and $c(\mathrm{LCV})$ is the diffusion tensor defined in (eq.9). The PDE (eq.4) is solved using an iterative Jacobi method. Time and space are discretized in the following way : $t=n \Delta t, n=0,1, . . x=i \eta, i=0,1, \ldots M-1$ and $y=j \eta, j=0,1, \ldots N-1$, with $M \eta * N \eta$ being the image size. At iteration $n+1$ we have:

$$
I_{i, j}^{n+1}=I_{i, j}^{n}+\frac{1}{4} \Delta t d_{i, j}^{n}
$$

where

$$
\begin{gathered}
d_{i, j}^{n}=\frac{1}{\eta^{2}}\left[R_{i+1, j}^{n}\left(I_{i+1, j}^{n}-I_{i, j}^{n}\right)+R_{i-1, j}^{n}\left(I_{i-1, j}^{n}-I_{i, j}^{n}\right)+\right. \\
\left.R_{i, j+1}^{n}\left(I_{i, j+1}^{n}-I_{i, j}^{n}\right)+R_{i, j-1}^{n}\left(I_{i, j-1}^{n}-I_{i, j}^{n}\right)\right], \\
R_{x}^{n}= \begin{cases}\frac{1}{2}\left[1-\Gamma^{n}(x)\right]^{2} & \text { if } \Gamma^{n}(x) \leq 1 \\
0 & \text { otherwise }\end{cases}
\end{gathered}
$$

where

$$
\Gamma^{n}(x)=\frac{\mathrm{LCV}_{e}^{2}(x ; n)-\mathrm{GCV}^{2}(n)}{\operatorname{GCV}^{2}(n)\left(1+\mathrm{GCV}^{2}(n)\right)} .
$$

$$
\begin{gathered}
\operatorname{LCV}_{e}^{2}(x ; n)=\frac{\left|\frac{1}{2}\left\|\nabla I_{i, j}^{n}\right\|^{2}-\frac{1}{16}\left(\nabla^{2} I_{i, j}^{n}\right)^{2}\right|}{\left[I_{i, j}^{n}+\frac{1}{4} \nabla^{2} I_{i, j}^{n}\right]^{2}} \\
\left\|\nabla I_{i, j}^{n}\right\|=\frac{1}{\sqrt{2}}\left(\left\|\nabla_{+} I_{i, j}^{n}\right\|^{2}+\left\|\nabla_{+} I_{i, j}^{n}\right\|^{2}\right)^{\frac{1}{2}} \\
\nabla_{ \pm} I_{i, j}^{n}= \pm \frac{1}{\eta}\left[I_{i \pm 1, j}^{n}-I_{i, j}^{n}, I_{i, j \pm 1}^{n}-I_{i, j}^{n}\right], \\
\nabla^{2} I_{i, j}^{n}=\frac{1}{\eta^{2}}\left[I_{i+1, j}^{n}+I_{i-1, j}^{n}+I_{i, j+1}^{n}+I_{i, j-1}^{n}-4 I_{i, j}^{n}\right],
\end{gathered}
$$

and

$$
\operatorname{GCV}(n)=\frac{h}{\sqrt{2}} M A D\left[\nabla \ln I_{i, j}^{n}\right]
$$

where $M A D$ stands for median absolute deviation and $h=1.4826$. The image $I_{i, j}^{n}$ is considered stationnary when $\operatorname{GCV}(n) \leq \epsilon$, a small positive threshold defined empirically. Figure (1) (b) shows result of this method. We then calculate the contour image

$$
I_{\mathrm{LCV}}(i, j)= \begin{cases}\operatorname{LCV}_{e}^{n}(i, j) & \text { if } \operatorname{LCV}_{e}^{n}(i, j)>\tau \\ 0 & \text { otherwise. }\end{cases}
$$

Figure 1 (c) shows an example of such image. The gradient amplitude of this image is used to compute the external energy $E_{\text {extern }}(u)$.

Considering the fact that the direction of the gradient is perpendicular to the edge (figure 2), we can improved the effectiveness of the external energy by forcing $\vec{\nabla} I_{\mathrm{LCV}}$ to align with $\vec{n}[19,8]$.

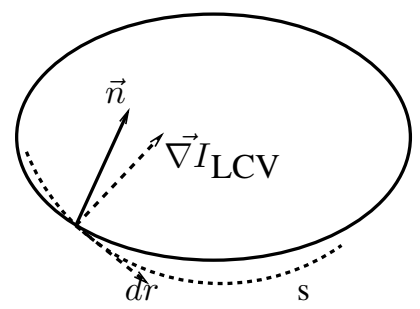

Figure 2. $I_{\mathrm{LCV}}$ gradient and the normal to the B-spline snake

Therefore we can write:

$$
E_{\text {extern }}(u)=-|\overrightarrow{\nabla I} \operatorname{LCV}(s(u)) \cdot(\vec{n}(s(u)))|
$$

where $\vec{n}(s(u))$ denotes the unit normal vector of the Bspline snake curve $s$.

\subsection{Curve evolution}

The optimal segmentation corresponds to the B-spline snake that minimizes the total energy :

$E_{s}=\left.\alpha \int_{0}^{1}|| s^{\prime}(u)\right|^{2}-\left.c\right|^{2} d u+(1-\alpha) \int_{0}^{1} E_{\operatorname{extern}}(u) d u$ 


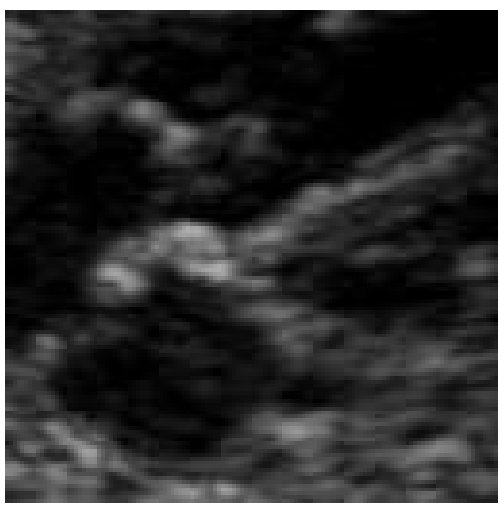

(a)

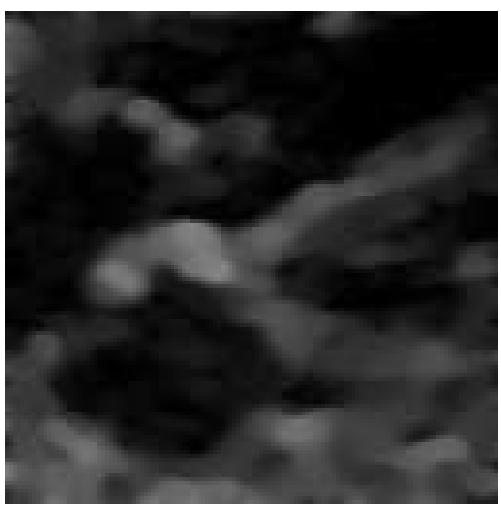

(b)

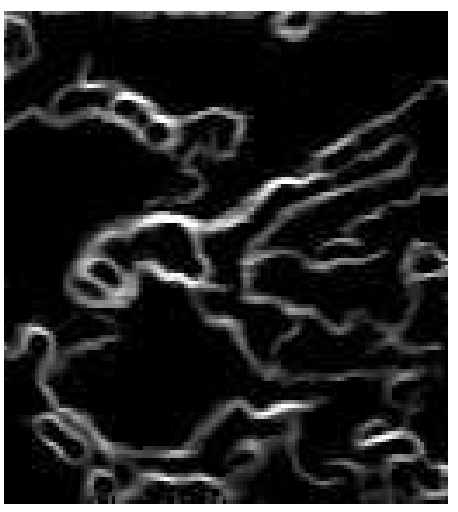

(c)

Figure 1. (a)Original image. (b)Diffusion filtered image using LCV and a robust tensor[14]. (c)Edge image using LCV.

where $\alpha$ is a parameter empirically defined, and $E_{s}$ is the total energy of $s$. We use the steepest descend algorithm to find that minimum. Let $T$ be the sampling rate of the curve and $L$ the number of knots, $L T$ points are sampled. For each iteration the total lenght of the curve is approximated by :

$$
\text { Length }(s)=\frac{1}{L} \sum_{i=0}^{L T-1} \sqrt{x^{\prime}\left(\frac{i}{T}\right)^{2}+y^{\prime}\left(\frac{i}{T}\right)^{2}}
$$

In order to control the global smoothness of the curve the distance $d_{k}$ between two knots is evaluated, $d_{k}=\frac{\text { Length }}{L}$. If $d_{k}$ is lower than a threshold $d_{\text {low }}$ a knot is added to decrease smoothness. If $d_{k}$ is higher than the threshold $d_{\text {high }}$ a knot is removed to increase smoothness.

\section{Results}

The model has been applied to an ultrasound video sequence of the four cardiac chambers view of a 12 weeks old fœetus. Figure 3 shows the results of the algorithm for different number of knots and different parameters. Figure 3 Image (a) show the original echographic image with the B-spline snake initialisation using 7 knots. In the same figure, images (b1), (b2), (c1), (c2) and (c3) show the segmentation results corresponding to the parameters shown in table 1. The number of knots in image (c3) was adaptively calculated by the algorithm. We notice that greater number of knots and a lower value of alpha lead to better results. However this tends to increase the computation time. A balanced number of knots (figure 3 (c2)) gives acceptable edge detection while keeping a low complexity.
Table 1. Results parameters

\begin{tabular}{lcc}
\hline \hline Figure & $\alpha$ & L \\
\hline b1 & 0.6 & fixed to 7 \\
b2 & 0.6 & fixed to 15 \\
c1 & 0.2 & fixed to 7 \\
c2 & 0.2 & fixed to 15 \\
c3 & 0.2 & adaptive \\
\hline \hline
\end{tabular}

\section{Discussion and conclusions}

In this paper we have presented our algorithm for a robust segmentation of echographic images using B-spline snakes. Unlike other approaches the external energy term uses the local coefficient of variation (LCV) to improve robustness to speckle. An edge image is created after an anisotropic diffusion step, based on the LCV gradient amplitude. The internal energy term forces the snakes to maintain a parameterization close to the curvilinear abscissa, and so ensures the smoothness of the curve. The experimental results on echocardiographic images are very encouraging. The limitation of this method is the significant number of parameters to be fixed by the user. We are currently investigating the automation of the algorithm.

\section{References}

[1] Kass M, Witkin A, Terzopoulos D. Snakes: Active contour models. Int J Comput Vis 1988;1:321-332.

[2] Cohen L. On active contour models and balloons. Computer vision graphics and image processing Image Understanding 1991;53:211-218.

[3] Delinguette H, Hebert M, Ikeuchi K. Shape representation and image segmentation using deformable surfaces. IEEE 


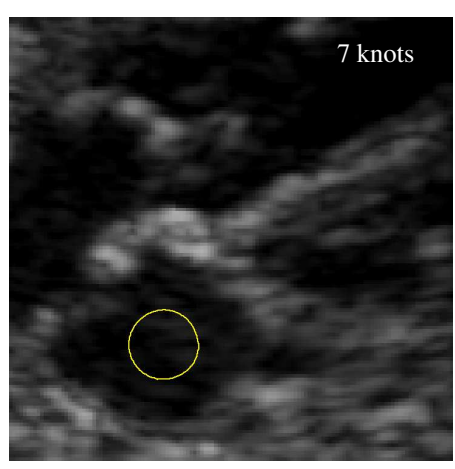

(a)

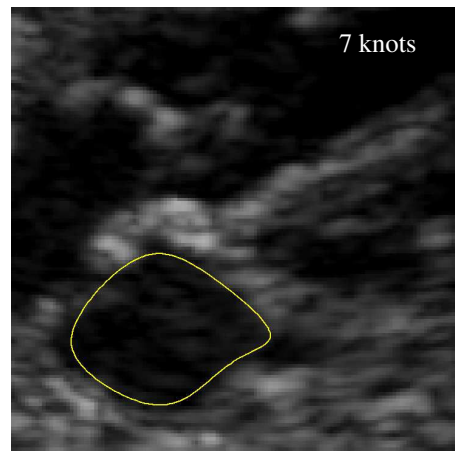

(c1)

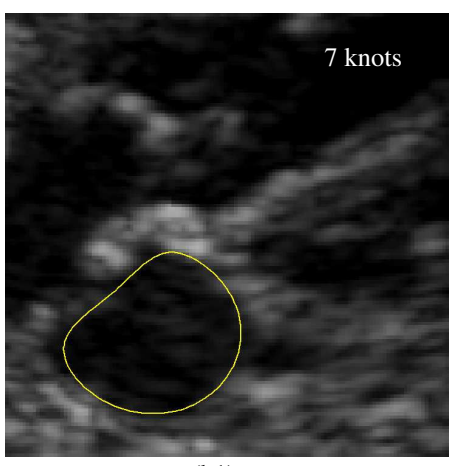

(b1)

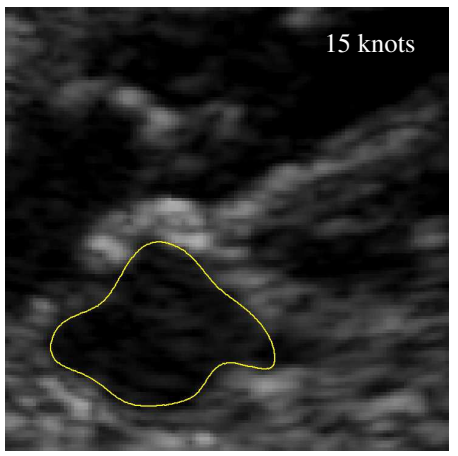

(c2)

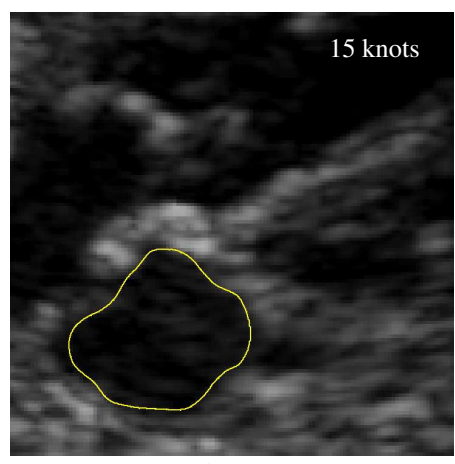

(b2)

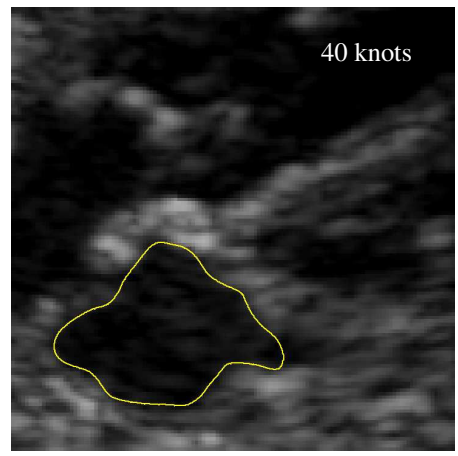

(c3)

Figure 3. Result of the proposed B-spline snake segmentation for different number of knots and parameters

Proceedings of Computer Vision and Pattern Recognition 1991;467-472.

[4] Davatzikos C, Prince J. An active contour model for mapping the cortex. IEEE Trans Med Imag 1995;14:6580.

[5] Menet S, Saint-Marc P, Medioni G. B-snakes: Implementation and application to stereo. Proc Image Understanding Workshop 1990;720-726.

[6] Mortensen E, Morse B, Barrett W, Udupa J. Adaptive boundary detection using 'live-wire' two-dimensional dynamic programming. Computers in Cardiology 1992; 635-638.

[7] Brigger P, Hoeg J, Unser M. B-spline snakes: A flexible tool for parametric contour detection. IEEE Trans Image Processing 2000;9:1484-1496.

[8] Jacob M, Blu T, Unser M. Efficient energies and algorithms for parametric snakes. IEEE Trans on Image Processing 2004;13:1231-1244.

[9] Cohen I, Ayache N, Sulger P. Tracking points on deformable objects using curvature information. ECCV Lectures Notes in Computer Science 1992;458-466.

[10] Cremers D, Schnorr C, Weickert J. Diffusionsnakes: combining statistical shape knowledge and image information in a variational framework. Proc IEEE Work on Variational and Level Set Methods 2001;137-144.

[11] Klein A, Egglin T, Pollak J, Lee F, Amini A. Identifying vascular features with orientation specific filters and bspline snakes. Computers in Cardiology 1994;113-116.

[12] Berger MO, Maurice N, Winterfeldt G, Lethor JP.
Automatic $3 \mathrm{~d}$ reconstruction of the beating left ventricle using transthoracic echographic images. Computers in Cardiology 1998;641-644.

[13] Xu C, Prince J. Snakes, shapes, and gradient vector flow. IEEE Trans on Image Proc 98;7:359-369.

[14] Tauber C, Batatia H, Ayache A. Speckle filtering with robust anisotropic diffusion. Proc Advanced Concepts for Intelligent Vision Systems 2004;

[15] Yu Y, Acton S. Speckle reducing anisotropic diffusion. IEEE Trans on Image Proc 2002;11:1260-1270.

[16] Schoenberg I. Cardinal spline interpolation. PA SIAM 1973;.

[17] Staib L, Duncan J. Boundary fitting with parametrically deformable models. IEEE Trans Pattern Anal Machine Intell 1992;14:1061-1075.

[18] Chakraborty A, Staib L, Duncan J. Deformable boundary finding in medical images by integrating gradient and region information. IEEE Trans Med Imaging 1996; 15:859-870.

[19] Park H, Schoepflin T, Kim Y. Active contour model with gradient directional information: Directional snake. IEEE Trans Circuits Syst Video Technol 2001;11:252-256.

Address for correspondence:

Clovis Tauber

2 rue camichel, BP7122. 31071 Toulouse Cedex 7, France tauber@enseeiht.fr 